\title{
Caracterização e perspectivas de gestantes em um serviço especializado de pré-natal
}

\author{
Characterization and perspectives of pregnant women in a specialized prenatal service \\ Caracterización y perspectivas de la gestante en un servicio prenatal especializado
}

Recebido: 15/07/2021 | Revisado: 20/07/2021 | Aceito: 21/07/2021 | Publicado: 28/07/2021

\author{
Laiane Cristine Silva Alvarenga \\ ORCID: https://orcid.org/0000-0003-0977-6086 \\ Pontifícia Universidade Católica de Minas Gerais, Brasil \\ E-mail: layanesilva757@gmail.com \\ Alessandra Carvalho dos Santos \\ ORCID: https://orcid.org/0000-0001-6590-2062 \\ Pontifícia Universidade Católica de Minas Gerais, Brasil \\ E-mail: lela.alessandra10@gmail.com \\ Patrícia Scotini Freitas \\ ORCID: https://orcid.org/0000-0002-8270-8955 \\ Universidade Federal de Alfenas, Brasil \\ Email: patricia.freitas@unifal-mg.edu.br \\ Munyra Rocha Silva Assunção \\ ORCID: https://orcid.org/0000-0002-0998-3125 \\ Universidade Federal de Alfenas, Brasil \\ Email: munyrarsilva@hotmail.com \\ Cristiane Aparecida Silveira-Monteiro \\ ORCID: https://orcid.org/0000-0002-8427-7220 \\ Universidade Federal de Alfenas, Brasil \\ Email: cristiane.monteiro@unifal-mg.edu.br
}

\begin{abstract}
Resumo
Objetivo: avaliar a perspectiva da gestante com relação à gestação e ao serviço de saúde especializado de pré-natal em uma cidade de Minas Gerais, bem como caracterizar o perfil e expectativas das gestantes atendidas. Método: estudo descritivo, quali-quantitativo que aconteceu em um serviço de assistência especializada. Os dados foram analisados a partir da estatística descritiva e Análise de Conteúdo (Bardin). Resultados: participaram 41 gestantes, sendo quatro $(9,8 \%)$ menores de 18 anos, três $(7,3 \%)$ acima dos 35; 13 (31,7\%) com hipertensão. A escolaridade apresentou associação com a participação de atividades educativas no pré-natal $\left(\chi^{2}(3)=6,258 ; \mathrm{p}=0,100 ; \mathrm{V}\right.$ de Cramer $\left.=0,391\right)$ e o número de dependentes e o mês de início das consultas $(\rho=0,356 ; p=0,031)$. As categorias foram: "Acolhimento, Segurança e Proteção", "Insegurança, Medo e Decepção"; "Indiferença" e "Expectativas". Conclusão: a percepção das gestantes mostra lacunas no cuidado, bem como crenças que podem interferir no acompanhamento gestacional, sendo necessário acolhimento e acompanhamento efetivo.
\end{abstract}

Palavras-chave: Gravidez de alto risco; Cuidado pré-natal; Saúde pública; Educação em saúde; Enfermagem.

\begin{abstract}
Objective: to evaluate the outlook of pregnant women regarding pregnancy and specialized prenatal care in a city in the State of Minas Gerais, and to characterize the profile and expectations of the pregnant women assisted. Method: this is a descriptive, quali-quantitative study carried out in a specialized care service. The data were analyzed using descriptive statistics and Content Analysis (Bardin). Results: 41 pregnant women participated in the study, four (9.8\%) of them were under 18 years, three $(7.3 \%)$ of them were over 35 years, and $13(31.7 \%)$ of them suffered from hypertension. The level of education was associated with participation in educational activities during prenatal care $\left(\chi^{2}(3)=6.258 ; p=0.100\right.$; Cramer's $\left.V=0.391\right)$ and the number of dependents and the month when the appointments started ( $\rho=0.356 ; p=0.031$ ). The categories were the following: "Welcoming, Safety, and Security"; "Insecurity, Fear and Disappointment"; "Indifference" and "Expectations". Conclusion: the perception of pregnant women indicates gaps in care, as well as beliefs that can interfere with following up on pregnancy, meaning that effective welcoming and follow-up procedures are necessary.
\end{abstract}

Keywords: High-risk pregnancy; Prenatal care; Public health; Health education; Nursing.

\section{Resumen}

Objetivo: evaluar la perspectiva de la gestante en relación al embarazo y al servicio de salud especializado prenatal en una ciudad de Minas Gerais, y caracterizar el perfil y las expectativas de las gestantes. Método: estudio descriptivo, cuali-cuantitativo desarrollado en servicio de atención especializada. Datos analizados a partir de estadística 
descriptiva y Análisis de Contenido (Bardin). Resultados: participaron 41 gestantes, siendo 4 (9,8\%) menores de 18 años; 3 (7,3\%) sobre 35 años; 13 (31,7\%) con hipertensión. La escolaridad estuvo relacionada con la participación en actividades educativas en el prenatal $\left(\chi^{2}(3)=6,258 ; p=0,100 ; \mathrm{V}\right.$ de Cramer $\left.=0,391\right)$, el número de dependientes y el mes de inicio de las consultas $(\rho=0,356 ; p=0,031)$. Las categorías fueron: "Acogida, Seguridad y Protección"; "Inseguridad, Miedo y Decepción"; "Indiferencia"; y "Expectativas". Conclusión: la percepción de las gestantes muestra lagunas en el cuidado, así como creencias que pueden interferir en el seguimiento gestacional, siendo necesarios la acogida y el seguimiento efectivo.

Palabras clave: Embarazo de alto riesgo; Cuidado prenatal; Salud pública; Educación en salud; Enfermería.

\section{Introdução}

Ainda que a gravidez seja um evento fisiológico, natural, uma série de condições clínicas, obstétricas ou sociais podem trazer complicações ao período gestacional caracterizando uma gestação de alto risco (Brasil, 2012a).

A avaliação do risco gestacional considera diversos fatores: características individuais e condições sociodemográficas desfavoráveis (menor de 17 e maiores que 35 anos, ocupações de risco, situação conjugal insegura, baixa escolaridade, altura menor que 1,45 m, peso menor que $45 \mathrm{~kg}$ ou maior que $75 \mathrm{~kg}$ e a dependência de drogas lícitas ou ilícitas); história reprodutiva anterior (morte perinatal, recém-nascido com crescimento retardado, pré-termo ou malformado, abortamento habitual, esterilidade ou infertilidade, intervalo temporal entre os partos menor que dois ou maior que cinco anos, nuliparidade ou multiparidade, síndrome hemorrágica ou doença hipertensiva e cirurgia uterina anterior); doenças obstétricas na gravidez atual (desvio quanto ao crescimento uterino, número de fetos e volume de líquidos amnióticos, trabalho de parto prematuro e gravidez prolongada, ganho ponderal inadequado, pré-eclâmpsia e eclâmpsia, amniorrexe prematura, isoimunização hemorragias de gestação e óbito fetal); intercorrências clínicas (neoplasias, cardiopatias, pneumopatias, nefropatias, endocrinopatias, hemopatias, hipertensão arterial, epilepsia, doenças infecciosas, doenças autoimune e ginecopatias) (Brasil, 2019). Ressalta-se que não são apenas aspectos biológicos, mas também fatores afetivos, comportamentais relacionados a problemas familiares e sociais que interferem no período gestacional trazendo consequências para a mãe e/ou lactente (Antoniazzi, Siqueira \& Farias, 2019).

O acompanhamento pré-natal é normalmente realizada pela Estratégia de Saúde da Família (ESF). Entretanto, quando constatadas situações de risco, a gestante é referenciada para o acompanhamento pré-natal especializado (Brasil, 2019). Ao serem encaminhadas para a atenção secundária podem surgir outras preocupações e receios, o que pode gerar problemas de ordem física e/ou psicológicas (Antoniazzi, Siqueira \& Farias, 2019). Apesar da percepção de risco na gravidez ser individual (Lee, Ayers \& Holden, 2016), tem sido demonstrado que ser rotulado como de alto risco pode aumentar a ansiedade (Taghizadeh et al., 2017).

Deste modo, o presente estudo tem o objetivo de avaliar a perspectiva da gestante com relação à gestação e ao serviço de saúde especializado de pré-natal em uma cidade de Minas Gerais, bem como caracterizar o perfil e expectativas das gestantes atendidas.

\section{Metodologia}

Trata-se de uma pesquisa quali-quantitativa, descritiva e exploratória. O estudo foi realizado em um Núcleo de Especialidade de um município do Sul de Minas Gerais.

No período do estudo (2017) o serviço de referência fazia o pré-natal especializado de 66 gestantes a qual constituía a população do estudo. Conforme o critério de exclusão, 20 participantes foram excluídas por faltarem à três consultas e cinco recusaram participar do estudo, resultando em uma amostra de 41 gestantes.

Para a coleta de dados utilizou-se um instrumento tipo formulário semiestruturado, construído pelos autores e refinado por especialistas. O instrumento continha duas partes: na primeira abordavam: condições sociodemográficas e de trabalho das 
mulheres; experiências com a gravidez e serviços e percepções sobre a gestação e com o serviço de saúde. Buscou-se estabelecer uma interação de confiança e respeito com as mulheres, por intermédio de conversas informais, escuta atenta, individualização das entrevistas, respostas às indagações e solicitações, e explicação da pesquisa, do interesse acadêmico, dos aspectos éticos envolvidos. A questão norteadora foi "Fale como foi para a senhora ser classificada como gravidez de risco e encaminhada para o núcleo de especialidade"?

As entrevistas foram realizadas de maio a julho de 2017, gravadas em aparelho de MP4, além de um diário de campo. A captação das mulheres foi realizada por conveniência no momento da espera pela consulta no próprio ambulatório. As gestantes foram abordadas, apresentadas à pesquisa e as que consentiram com a participação, após a assinatura de Termo de Consentimento Livre e Esclarecido (TCLE), foram convidadas a um local reservado para responder à entrevista.

Os dados qualitativos foram analisados segundo a Análise de Conteúdo (Bardin, 2010), norteada pelo objetivo da pesquisa. Organizou-se o material, fez-se a leitura/releitura do mesmo e se assinalou, além de sentidos apreendidos do todo do material, os temas ou unidades de significados de interesse. Considerou-se na análise, as mensagens explícitas e implícitas. $\mathrm{Na}$ sequência, os temas foram agregados por similaridades, classificados e reclassificados e, serviram de base à inferência apoiada também na teoria.

Para garantia do anonimato, a identificação dos sujeitos foi realizada por nomes de flores seguido do número da ordem de realização das entrevistas. Não foi realizada a correção gramatical ou ortográfica das falas das entrevistadas.

Para os dados quantitativos foi construído um banco de dados com dupla alimentação para posterior análise estatística utilizando-se o programa Statistical Package for the Social Sciences (SPSS), versão 21.0. Foi realizada a estatística descritiva e inferencial utilizando-se o teste t de amostras independentes, o Kruskal-Wallis, correlação de Spearman e quiquadro.

O estudo recebeu Parecer (1.956.960/2017) favorável do Comitê de Ética em Pesquisa em Seres Humanos da Pesquisa da Pontifícia Universidade Católica de Minas Gerais. Foram respeitados os preceitos éticos de participação voluntaria e consentida de cada sujeito (Brasil, 2012b).

\section{Resultados}

Foram pesquisadas 41 gestantes cujos dados biopsicossociais são apresentados na Tabela 1. 
Tabela 1 - Distribuição das gestantes com acompanhamento especializado segundo dados biopsicossociais. Minas Gerais, $2017(n=41)$.

\begin{tabular}{|c|c|c|c|}
\hline \multirow{2}{*}{ Variáveis } & & \multicolumn{2}{|c|}{ Total } \\
\hline & & f & $\%$ \\
\hline \multirow{6}{*}{ Faixa etária } & Menores de 18 anos & 12,20 & 12,20 \\
\hline & 18 a 23 anos & 26,83 & 26,83 \\
\hline & 24 a 29 anos & 24,39 & 24,39 \\
\hline & 30 a 35 anos & 19,51 & 19,51 \\
\hline & Acima de 35 anos & 17,07 & 17,07 \\
\hline & Total & 41 & 100,0 \\
\hline \multirow{4}{*}{ Grau de Escolaridade } & Ensino Fundamental & 9 & 22,0 \\
\hline & Ensino Médio & 26 & 63,4 \\
\hline & Ensino Superior & 6 & 14,6 \\
\hline & Total & 41 & 100,0 \\
\hline \multirow{3}{*}{ Estado Civil } & Com companheiro & 28 & 68,3 \\
\hline & Sem companheiro & 13 & 31,7 \\
\hline & Total & 41 & 100,0 \\
\hline \multirow{5}{*}{ Religião } & Cristãs & 32 & 78,0 \\
\hline & Espírita & 3 & 7,3 \\
\hline & Nenhuma & 2 & 4,9 \\
\hline & Outras & 4 & 9,8 \\
\hline & Total & 41 & 100,0 \\
\hline \multirow{4}{*}{ Raça/etnia } & Branca & 25 & 61,0 \\
\hline & Negra/ Parda & 15 & 36,6 \\
\hline & Não relatou & 1 & 2,4 \\
\hline & Total & 41 & 100,0 \\
\hline \multirow{6}{*}{ Profissão } & Trabalhadores dos serviços domésticos & 25 & 61,0 \\
\hline & Operadores do comércio & 5 & 12,2 \\
\hline & Agentes administrativos & 4 & 9,8 \\
\hline & Outras & 6 & 14,6 \\
\hline & Nenhum & 1 & 2,4 \\
\hline & Total & 41 & 100,0 \\
\hline \multirow{6}{*}{ Renda Familiar } & Até 1 salário-mínimo & 3 & 7,3 \\
\hline & Entre 1 e 2 salários & 25 & 61,0 \\
\hline & Entre 3 e 4 salários & 11 & 26,9 \\
\hline & Recusou-se a responder & 1 & 2,4 \\
\hline & Sem renda no momento & 1 & 2,4 \\
\hline & Total & 41 & 100,0 \\
\hline \multirow{4}{*}{$\mathbf{N}^{\circ}$ de dependentes } & 1 a 2 & 9 & 21,9 \\
\hline & 3 a 4 & 25 & 61,0 \\
\hline & 5 ou mais & 7 & 17,1 \\
\hline & Total & 41 & 100,0 \\
\hline
\end{tabular}


Em relação ao perfil, cinco (12,2\%) gestantes menores de 18 anos de idade e sete $(17,1 \%)$ acima de 35 anos; 26 $(63,4 \%)$ possuem ensino médio, $22(78,0 \%)$ são cristãs, $25(61,0 \%)$ se declaram brancas e $28(68,3 \%)$ possuem companheiros.

Quanto aos aspectos profissionais e econômicos: 25 (61\%) relataram ser trabalhadores dos serviços domésticos em geral; $25(61 \%)$ têm renda familiar entre 1 e 2 salários; 25 (61\%) possuem de 3 a 4 dependentes e $26(63,4 \%)$ relataram ser 1 pessoa apenas que contribui para a renda familiar.

Na Tabela 2 foram considerados dados de saúde e gestação.

Tabela 2 - Distribuição de Gestantes Segundo Dados de Saúde e Gestação. Minas Gerais, 2017 (n=41).

\begin{tabular}{|c|c|c|c|}
\hline \multirow{2}{*}{ Variáveis } & & \multicolumn{2}{|c|}{ Total } \\
\hline & & $\mathbf{f}$ & $\%$ \\
\hline \multirow{6}{*}{$\begin{array}{l}\text { Usava quando engravidou como } \\
\text { método contraceptivo }\end{array}$} & Nenhum & 22 & 53,6 \\
\hline & Anticoncepcional oral combinado & 7 & 17,1 \\
\hline & Camisinha masculina & 5 & 12,2 \\
\hline & Anticoncepcional injetável & 4 & 9,8 \\
\hline & Combinação de 2 métodos & 3 & 7,3 \\
\hline & Total & 41 & 100,0 \\
\hline \multirow{4}{*}{ Essa gestação foi } & Desejada e planejada & 17 & 41,5 \\
\hline & Desejada, mas não planejada & 17 & 41,5 \\
\hline & Não desejada, nem planejada & 7 & 17,0 \\
\hline & Total & 41 & 100,0 \\
\hline \multirow{12}{*}{ Motivos de encaminhamento } & Hipertensão & 13 & 31,7 \\
\hline & Diabetes Mellitus & 4 & 9,8 \\
\hline & Adolescente & 4 & 9,8 \\
\hline & Acima de 35 anos & 3 & 7,3 \\
\hline & Sífilis & 2 & 4,9 \\
\hline & Hipotireoidismo & 1 & 2,4 \\
\hline & Hepatite C & 1 & 2,4 \\
\hline & Cardiopata & 1 & 2,4 \\
\hline & Toxoplasmose & 1 & 2,4 \\
\hline & Usuário de substâncias ilícitas & 1 & 2,4 \\
\hline & Outras condições & 10 & 24,5 \\
\hline & Total & 41 & 100,0 \\
\hline \multirow{10}{*}{ Condições associadas } & Sem condições associadas & 31 & 75,8 \\
\hline & Trombose & 2 & 4,9 \\
\hline & Gemelaridade & 2 & 4,9 \\
\hline & Diabetes Mellitus & 1 & 2,4 \\
\hline & Oligodrâmnio & 1 & 2,4 \\
\hline & Sífilis & 1 & 2,4 \\
\hline & Foco & 1 & 2,4 \\
\hline & Usuário de substância ilícitas & 1 & 2,4 \\
\hline & RH negativo & 1 & 2,4 \\
\hline & Total & 41 & 100,0 \\
\hline Antecedentes obstétricos: Parto & Nenhum parto & 16 & 39,1 \\
\hline
\end{tabular}




\section{Antecedentes obstétricos: Natimorto/Aborto}

Antecedentes obstétricos: Gestações

\section{Semana que iniciou o pré-natal}

Atividades educativas que participou*

\section{Quantas consultas realizou até agora}

$\begin{array}{ll} & \text { Acima de } 9 \text { consultas } \\ & \text { Total } \\ & \text { Cuidados de higiene } \\ & \text { Aleitamento materno } \\ & \text { Prevenção a Infeções Sexualmente Transmissíveis } \\ & \text { IST) } \\ & \text { Modificações corporais, emocionais durante a } \\ & \text { gestação } \\ \text { Atividades educativas que participou* } & \text { Importância do pré-natal } \\ & \text { Preparo para o parto } \\ & \text { Orientação nutricional } \\ & \text { Etiopatogenia das Infeções Sexualmente } \\ & \text { Transmissíveis } \\ & \text { Desenvolvimento gestacional } \\ & \text { Orientação sobre fumo, álcool e drogas } \\ & \text { Planejamento familiar }\end{array}$

* A participante poderia marcar mais de uma alternativa.

Fonte: dados da pesquisa (2017).

Quanto ao uso de métodos quando engravidou: 22 (53,6\%) relataram que não usavam nenhum método; 17 (41,5\%) relataram que foi uma gestação desejada e planejada e em igual valor que foi desejada, mas não planejada.

Em relação ao motivo de encaminhamento: 13 (31,7\%) foram diagnosticadas com hipertensão, quatro $(9,8 \%)$ com diabetes mellitus, quatro $(9,8 \%)$ adolescente, três (7,3\%) acima de 35 anos, duas (4,9\%) sífilis, 1 (2,4\%) com hipotireoidismo, 
hepatite C, cardiopatia, toxoplasmose, usuária de substâncias ilícitas, respectivamente e por fim $10(24,4 \%)$ apresentavam outras doenças como: hemorroidas, cirurgia bariátrica, dores abdominais, colo uterino pequeno, sobrepeso, dentre outras. Do total, 31 (75,8\%) não tem condições associadas. Em relação às complicações em gestações anteriores: sete $(17,1 \%)$ a mesma complicação dessa gestação.

Quanto aos antecedentes obstétricos: $16(39,1 \%)$ são primíparas, nove $(21,9 \%)$ tiveram abortos anteriores e seis $(14,7 \%)$ natimortos. Do total, 19 (46,3\%) iniciaram o pré-natal em até 4 semanas de gestação e 18 (43,9\%) tiveram de 4 consultas até o momento da entrevista.

Com relação às atividades educativas recebidas, 20 (48,8\%) receberam orientações sobre cuidados de higiene e 19 $(46,3 \%)$ sobre aleitamento materno.

Em relação aos testes de associação das variáveis, foi observada uma tendência de associação estatisticamente significativa entre a faixa etária e realização do planejamento reprodutivo $\left(\chi^{2}(2)=5,539 ; p=0,063 ; \mathrm{V}\right.$ de Cramer $\left.=0,368\right)$, sendo que aquelas com menos de 18 tendem a estar associadas com maior frequência a não ter participado de ações, enquanto aquelas entre 24 e 35 anos tendem a estar mais frequentemente associadas a ter participado de ações.

Foi observada uma tendência de associação estatisticamente significativa entre a escolaridade e a participação de ações educativas $\left(\chi^{2}(3)=6,258 ; \mathrm{p}=0,100 ; \mathrm{V}\right.$ de Cramer $\left.=0,391\right)$, de preparo para o parto $\left(\chi^{2}(3)=7,793 ; \mathrm{p}=0,050 ; \mathrm{V}\right.$ de Cramer $=0,436)$, de orientação nutricional $\left(\chi^{2}(3)=11,089 ; \mathrm{p}=0,011 ; \mathrm{V}\right.$ de Cramer $\left.=0,520\right)$, de conscientização da importância do pré-natal $\left(\chi^{2}(3)=7,793 ; \mathrm{p}=0,050 ; \mathrm{V}\right.$ de Cramer $\left.=0,436\right)$, de importância do aleitamento materno $\left(\chi^{2}(3)=\right.$ 8,588; $\mathrm{p}=0,035 ; \mathrm{V}$ de Cramer $=0,458$ ), sendo que aquelas com ensino fundamental tendem a estar mais frequentemente associadas a não terem participado, enquanto aquelas com ensino superior apresentam a relação inversa.

A escolaridade também apresentou tendência de associação estatisticamente significativa sendo atividades de prevenção e sexualidade $\left(\chi^{2}(3)=7,113 ; p=0,068 ; \mathrm{V}\right.$ de Cramer $\left.=0,417\right)$ e orientações sobre IST $\left(\chi^{2}(3)=8,085 ; \mathrm{p}=0,044 ; \mathrm{V}\right.$ de Cramer $=0,444$ ), uma vez que aquelas com ensino superior tendem a estar mais frequentemente associadas a ter participado das atividades.

Foi observada associação estatisticamente significativa entre o estado civil e orientações sobre o desenvolvimento gestacional $\left(\chi^{2}(2)=6,322 ; \mathrm{p}=0,042 ; \mathrm{V}\right.$ de Cramer $\left.=0,393\right)$, sendo que ser solteira está mais frequentemente associado a não ter comparecido às orientações. Um aumento no tamanho da amostra talvez destaque uma relação inversa a esta para a condição de casada.

Constatou-se uma tendência de associação estatisticamente significativa entre o estado civil e orientações sobre aleitamento materno $\left(\chi^{2}(2)=5,126 ; \mathrm{p}=0,077 ; \mathrm{V}\right.$ de Cramer $\left.=0,354\right)$, sendo que estar sem companheiro está mais frequentemente associado a não ter participado de orientação sobre amamentação e menos associado a ter tido. Um aumento da amostra pode destacar a relação inversa para as casadas.

Também constatou-se associação estatisticamente significativa entre atividade remunerada e atividades de conscientização sobre a importância do pré-natal $\left(\chi^{2}(1)=3,939 ; \mathrm{p}=0,047 ; \mathrm{V}\right.$ de Cramer $\left.=0,310\right)$, desenvolvimento gestacional $\left(\chi^{2}(1)=3,930 ; p=0,047 ; \mathrm{V}\right.$ de Cramer $\left.=0,310\right)$, cuidados com higiene $\left(\chi^{2}(1)=2,930 ; p=0,087 ; \mathrm{V}\right.$ de Cramer $\left.=0,267\right)$, preparos do parto $\left(\chi^{2}(1)=3,939 ; \mathrm{p}=0,047 ; \mathrm{V}\right.$ de Cramer $\left.=0,310\right)$, orientação nutricional $\left(\chi^{2}(1)=3,939 ; \mathrm{p}=0,047 ; \mathrm{V}\right.$ de Cramer $=0,310)$, orientações quanto ao fumo, álcool e drogas $\left(\chi^{2}(1)=3,939 ; \mathrm{p}=0,047 ; \mathrm{V}\right.$ de Cramer $\left.=0,310\right)$, aleitamento materno $\left(\chi^{2}(1)=4,027 ; \mathrm{p}=0,045 ; \mathrm{V}\right.$ de Cramer $\left.=0,313\right)$, atividades sexuais e prevenções $\left(\chi^{2}(1)=4,027 ; \mathrm{p}=0,045 ; \mathrm{V}\right.$ de Cramer $=0,313)$, IST $\left(\chi^{2}(1)=3,939 ; \mathrm{p}=0,047 ; \mathrm{V}\right.$ de Cramer $\left.=0,310\right)$, em que a inserção nas ações está mais frequentemente 
associado a ter uma atividade remunerada e não participar de ações está mais associado a não ter uma atividade remunerada. Não foi observada associação estatisticamente significativa entre as demais variáveis.

Em relação aos testes de comparação, foi observada uma correlação positiva entre as variáveis número de dependentes da renda e o mês que iniciou as consultas $(\rho=0,356 ; p=0,031)$, sendo que quanto maior o número de dependentes, mais tardio foi o início das consultas.

Quanto à questão norteadora "Fale como foi para a senhora ser classificada como gravidez de risco e encaminhada para o núcleo de especialidade" emergiram quatro categorias: "Acolhimento, Segurança e Proteção", "Insegurança, Medo e Decepção"; "Indiferença" e "Expectativas".

\section{Categoria 1- Acolhimento, Segurança e Proteção}

Pode-se observar que em algumas falas, apesar das enormes mudanças atribuídas ao risco gestacional, algumas dessas gestantes relataram que um bom acolhimento e apoio eficaz das redes de saúdes, amenizam as inquietações vivenciadas durante esse período como também a necessidade de expressar seus sentimentos, e que muitas vezes não são ouvidas pelos profissionais de saúde.

O fato de se tratar de um serviço secundário de saúde também gera-lhes um sentimento de segurança, podendo ser identificadas nas falas a seguir:

Ao ser encaminhada para o núcleo me senti segura, pois há uma grande preocupação da área da saúde em relação a gestantes de alto risco [...] (Orquídea 4).

Eu acho que está muito bom, [...] vamos supor, a Doutora pega no meu pé mesmo, porque tem muita gente que tem gravidez de risco mais não leva a sério né e ela (médica) não. A cada dois anos você vai fazer esse exame, eu quero ver, e aí se não trouxer... risadas. Mais eu acho que o atendimento está sendo muito bom em relação a isso (Jasmim 19).

\section{Categoria 2- Insegurança, Medo e Decepção}

Pode-se observar que as gestantes demonstravam medo quanto ao acompanhamento, aos riscos para ela e para o bebê e às complicações que poderiam influenciar na gestação, especialmente se conseguiriam levá-la até o final.

Algumas gestantes alegaram não sentir confiança no que diz respeito ao atendimento, o que evidencia a falha em relação ao acolhimento individual dessas gestantes, podendo ser identificadas nos seguintes depoimentos:

Me assustei né, acreditava que no posto (ESF) teria atendimento com especialista, fiquei ansiosa e preocupada. E estou com medo do parto normal, ser forçada a algum procedimento de risco (Hortência 38).

Foi meio assustador né, quem achava que iria passar por uma gestação tranquila, é... não vai ser. (Amarílis 16).

\section{Categoria 3- Indiferença}

Algumas das gestantes não estavam envolvidas ao acompanhamento pré-natal, o que se supõe que, devido à falha na atenção dos profissionais além do não esclarecimento do diagnóstico repercutia na não adesão e no vínculo com sistema de saúde. Algumas mulheres demonstraram tal sentimento com relação ao pré-natal, como observado na seguinte fala: 
Ah eu não sei porque um dia me ligaram e falaram que eu ia vir pra cá agora e que ia ser na Zona Leste por causa da minha gravidez ser de risco, mas tanto faz [...] (Violeta 31).

\section{Categoria 4 - Expectativas}

Por fim, pode-se constatar que as gestantes entrevistadas acreditam na capacidade e na importância do serviço especializado, relatando seus anseios para a possibilidade de melhora do serviço de pré-natal, identificados no seguinte depoimento:

\section{[...] é que só a médica mude com a gente porque pela minha primeira gravidez eu acho assim, que eu tinha muitas} dúvidas e ela em nenhum momento [...] me deixou calma, ela só colocou bobeira na minha cabeça [...] eu espero [...] que eles possam por um médico sim, profissional sim, mas primeiro humano, porque é isso que eu espero (Iris 34).

\section{Discussão}

O perfil das gestantes atendidas no serviço especializado de pré-natal foi: jovens, com ensino médio, com companheiro, cristãs, brancas, trabalhadoras dos serviços domésticos e que não participam de atividades de planejamento reprodutivo e consequentemente, engravidaram sem planejar.

A ausência de planejamento reprodutivo ainda é uma grave realidade brasileira, visto que pessoas com maior nível educacional e maior acessibilidade ao serviço de saúde são mais esclarecidas sobre o real significado do acompanhamento e preparação familiar do que aquelas com um baixo nível educacional. Isso se deve a uma falha do método de educação em saúde, além do modo de lidar com a individualidade, acarretando problemas no planejamento reprodutivo assim como, no cuidado consigo mesmo e na melhoria da qualidade de vida, já que este não envolve apenas métodos contraceptivos (Grein et al., 2017). Além disso, percebe-se que o planejamento reprodutivo ainda é fragmentado. Nesse estudo, 19 (46,3\%) gestantes usavam métodos contraceptivos e ainda sim, engravidaram.

Percebe-se este problema particularmente dentre adolescentes (Costa et al., 2016), como visto no presente estudo. A baixa escolaridade e o nível de educação limitado acarretam menor acesso às informações, considerado como um fator proporcional. Nessas adolescentes a gravidez não foi planejada, nem desejada ensejando diversos desafios: além do risco gestacional, as dificuldades para lidar com as mudanças sociais causadas por uma gravidez precoce (Rodrigues, Barros \& Soares, 2016). Apesar da maioria das gestantes possuírem o ensino médio no presente estudo, ainda assim apresentam problemas relacionados à participação no planejamento reprodutivo.

A carência de planejamento reprodutivo pode gerar sentimentos negativos com relação à gestação, principalmente se tratando de gestantes adolescentes sem uma preparação física e/ou psicológica para este período, trazendo um sentimento de indiferença com relação ao pré-natal e gravidez (Rodrigues, Barros \& Soares, 2016), observado também no presente estudo. A vulnerabilidade (Costa et al., 2016), pode desencadear em alterações mentais, o que traz danos ao desenvolvimento psíquico do feto já que este possui registros de memória, ou seja, afeta o vínculo do binômio.

Esse desinteresse geralmente é menos provável em mulheres acima de 35 anos, visto que existe uma maturidade com relação a gestação (Rodrigues, Barros \& Soares, 2016). Apesar de demonstrar afeição pela gestação e pré-natal, as mulheres de 35 anos ou mais que participaram da pesquisa relataram ainda não planejamento para a gestação atual.

Além das questões biológicas individuais, a situação conjugal está diretamente associada à segurança e estabilidade da mulher, tornando-se uma dificuldade para aquelas sem companheiro (Brasil, 2019). Não só a ausência do companheiro, mas especialmente a falta de apoio do pai da criança está associado a depressão pós-parto da mãe (Arrais; Araujo \& Schiavo, 2018). 
A religiosidade é fator importante para o enfrentamento de uma gestação de alto risco (Silva et al., 2020), já que estas estão sujeitas a desenvolverem um desgaste psicológico maior. A religião proporciona um alívio sobre a situação, tornando-as mais esperançosas em relação às dificuldades. Destaca-se também o papel da enfermagem pelo cuidado humanizado promovendo bem-estar e esclarecimento a essas gestantes (Dias et al., 2018). No presente estudo, pode-se observar que a maioria das entrevistadas possui uma crença religiosa, prevalecendo a cristã 32 (78\%).

A cor da gestante pode influenciar no aparecimento de fatores de risco bem como a morte materna quando se tratando da cor preta e parda (Costa et al., 2016; Martins \& Silva, 2018). Apesar da maioria branca, as pardas e pretas aparecem no presente estudo com suas singularidades e fatores de risco.

Constatou-se no presente estudo, que as gestantes que possuíam um número maior de dependentes, ou seja, que já tinham outros filhos, iniciavam o pré-natal mais tardiamente e as gestantes que tinham atividade remunerada participavam com mais frequência de ações educativas, o que é observado também em um estudo de base hospitalar realizado com 23894 mulheres entre 2011 e 2012, onde foi analisado uma menor adequação ao pré-natal entre mulheres multíparas, sem companheiro e sem trabalho remunerado (Domingues et al., 2015). Supõe-se que, experiências maternas lhe trariam mais segurança para lidar com gestações posteriores, consequentemente as mesmas não procuravam o pré-natal já no início.

Quanto aos aspectos profissionais e econômicos, no presente estudo pode-se perceber um baixo nível socioeconômico entre essas gestantes, pois $25(61,1 \%)$ declararam renda familiar entre 1 e 2 salários. A situação econômica pode estar associada à acessibilidade de informações e recursos ao longo do período gestacional, tornando-as mais suscetíveis a problemas no decorrer da gestação, colocando a equipe de saúde como o centro acolhedor durante esse período, principalmente a ESF (Brasil, 2012a).

O protocolo para encaminhamento obstétrico seleciona diversos problemas destacando a anemia grave, história de tromboembolismo, acretismo placentário, hipertireoidismo e outras comorbidades maternas graves. No entanto, é responsabilidade da equipe de saúde decidir pelo encaminhamento conforme necessidade da paciente (Salazar et al., 2016). No presente estudo, os principais diagnósticos encaminhados ao pré-natal especializado foram a hipertensão arterial e diabetes mellitus o que entra em consonância aos encontrados na literatura (Jantsch et al., 2017; Martins, Ghersel \& Ghersel, 2017), já que a maior incidência de óbitos entre gestantes está relacionada à complicações decorrentes da HAS e DMG (Jantsch et al., 2017).

Além dos diagnósticos médicos, mulheres diagnosticadas com gravidez de alto risco vivenciam e expressam vários eventos biológicos, comportamentais, afetivos, entrelaçados a situações familiares, institucionais e sociais, que extrapolam aqueles problemas diagnosticados, com possíveis consequências adversas à sua saúde e a de seu filho. Ou seja, enfrentam situações que comprometem ou podem afetar a sua saúde, incluindo o seu processo reprodutivo e as suas possibilidades de cuidar de si (Antoniazzi, Siqueira \& Farias, 2019). Tais sentimentos e situações também podem ser encontrados no presente estudo, no que diz respeito as perspectivas dessas gestantes enfatizados em suas falas.

Observou-se no presente estudo que alguns encaminhamentos poderiam ser evitados, pois, não existe real motivo comprovado de complicações, como por exemplo hemorroida, sobrepeso e bariátrica que ocupariam possivelmente uma vaga no sistema daquelas que necessitam de um atendimento especializado e com mais urgência (Brasil, 2012a). Com relação à referência de gestantes com bariátrica, há um protocolo municipal ao qual se considera um fator de risco e o possível acompanhamento pré-natal especializado, o que entra em divergência com as indicações do Ministério da Saúde (Brasil, 2012a). Indaga-se também como é a comunicação profissional-paciente, visto que grande parte dessas gestantes não sabiam o real motivo do encaminhamento para um centro de especialidade. 
Outro fator importante a ser analisado como um condicionante para um pré-natal especializado é a história reprodutiva anterior. Em um estudo feito em Fortaleza em 2016 com 68 gestantes classificadas como de risco 41, 2\% possuíram intercorrências em gestações anteriores (Rodrigues et al., 2017), um número bem aproximado ao presente estudo.

A adesão ao acompanhamento pré-natal e tratamento necessita de um vínculo entre o sistema de saúde e o cliente, esclarecimento de dúvidas e problemas que possam aparecer ao longo dessas 41 semanas, para que esse, se torne efetivo e mantenha qualidade nas suas ações (Pereira et al., 2018). Deve ser iniciado no primeiro trimestre, realizando por no mínimo seis consultas intercaladas entre médico e enfermeiro. Sempre que possível, as consultas devem ser realizadas mensalmente até 28. ${ }^{a}$ semana, quinzenalmente da $28 .^{a}$ até a $36 .^{a}$ semana e semanalmente da $36{ }^{a}$ até a $41 .^{a}$ semana (Brasil, 2012a).

A maioria das gestantes estava entre a $14 .^{a}$ e $26 .^{a}$ semana de gestação, o que pode indicar relação entre a gestação e a comorbidade como hipertensão crônica e diabetes gestacional, necessitando identificar os fatores de risco e realizar o acompanhamento efetivo para prevenção de possíveis complicações (Brasil, 2012a).

A identificação do risco deve ser realizada na primeira consulta e/ou nas subsequentes. É responsabilidade da equipe de pré-natal dar as informações sobre a maternidade de referência e anotar no cartão da gestante caso ocorra alguma intercorrência ao longo do período (Universidade Estadual de Campinas, 2017). Tanto o enfermeiro como o médico são responsáveis pelo atendimento (Menezes et al., 2020) e descoberta de um agravo, tornando então o possível encaminhamento para um pré-natal especializado. O possível agravo no quadro gestacional deve ser um alerta para a prestação de cuidados, no entanto, não se caracteriza pela transferência de cuidado (Universidade Estadual de Campinas, 2017), mesmo quando ocorra o encaminhamento, a ESF deve acompanhar todo o quadro dessa gestante mantendo o vínculo e o cuidado horizontal (Ragagnin et al., 2017).

Essas pacientes sentem-se seguras e protegidas quando são encaminhadas para o serviço de referência, pois acreditam que este possui melhores condições de modo a proporcionar suporte a gestação e um acompanhamento intensivo como visto nos depoimentos.

Esse julgamento dos serviços de pré-natal resulta da forma como as mulheres percebem, dentre outros aspectos, o que cabe-lhes, com base em ações, recursos e respostas que neles obtêm, no contato com a leitura médico-científica, reinterpretado, por outras leituras e, oportunidades educativas críticas e de exercício de direitos (Oliveira \& Mandú, 2015). Pode também refletir a visão medicalizada e especializada em que se valoriza, em especial, ações clínicas de controle curativo. Ao ter uma boa orientação e vínculo dos profissionais com essas pacientes, os indicadores de desempenho melhoram (Fernandez et al., 2020).

O fato de vivenciarem um sentimento negativo acerca do esclarecimento terapêutico, essas pacientes declararam que em virtude da falta de diálogo com profissionais de saúde, muitas possuíam dúvidas que geralmente não eram esclarecidas, ou ainda, eram sanadas com a mídia e fontes não confiáveis de informações, levando até mesmo um possível abandono do prénatal. Como observado em outros estudos o apoio e vínculo com os profissionais o esclarecimento das dúvidas e as informações sobre o encaminhamento e tratamento nesse período, fazem com que sejam amenizados tais sentimentos negativos, além de proporcionarem uma autonomia no próprio cuidado (Oliveira \& Mandú, 2015).

$\mathrm{Na}$ assistência do pré-natal é possível perceber muitas lacunas com relação ao atendimento oferecido pelos profissionais de saúde que muitas vezes se tornam mecanicista, deixando o cuidado humanizado a desejar, seja pela falta de empatia, escassez de informações e até mesmo com um tratamento grosseiro trazendo a essas gestantes um sentimento de insegurança, medo do processo gestacional e decepção em relação ao serviço (Ragagnin et al., 2017) como relatado por algumas gestantes no presente estudo.

Cabe ao enfermeiro e a equipe multiprofissional, orientá-las quanto suas dúvidas pessoais, suas necessidades, identificar possíveis dificuldades a adaptação ao tratamento, as restrições necessárias e aos procedimentos. Entende-se que, 
todo o pré-natal não deve ser um acolhimento voltado apenas a limitação patológica, mas também as necessidades emocionais, visto que essas possuem influência direta no seu estado de saúde além do apego maternal no pós-parto (Errico et al., 2018).

É importante frisar que fatores negativos da assistência refletem-se de modo biográfico para estas mulheres, além de apresentar de forma muito evidente a frieza e descaso de alguns profissionais ao atender as gestantes (Gaíva, Palmeira \& Mufato, 2017).

As expectativas com relação ao serviço de saúde se dá através da assistência recebidas por essas mulheres durante todo o pré-natal (Ragagnin et al., 2017). Apesar das dificuldades relatadas pelas gestantes do presente estudo, algumas destacam a dimensão do cuidado, que pode ser prestado pela equipe de saúde. Identifica que há qualidades profissionais no atendimento, contudo ainda necessita de um aprimoramento no que se diz atendimento humanizado e acolhedor correspondendo as suas necessidades.

Como citado anteriormente, a posição dos profissionais de saúde frente ao atendimento dessas gestantes não atende as expectativas, principalmente com relação ao papel da enfermagem já que esse em nenhum momento foi citado como acolhedor ou educador, ou seja, o médico foi quem orientou. Com isso percebe-se um distanciamento do enfermeiro diante do seu papel de educador em saúde na assistência especializada (Coelho et al., 2017).

Como limitações do estudo, destaca-se que os resultados aqui apresentados revelam percepções apenas de um dos envolvidos no processo assistencial à gravidez de alto risco e quanto ao próprio tamanho amostral. Sugerem-se outras pesquisas que abordem a perspectiva dos profissionais tanto da atenção primária quanto do serviço especializado, além dos próprios gestores.

A perspectiva de ouvir o sujeito do cuidado e que demanda a atenção dos serviços de saúde vem acrescentar conhecimento na área do pré-natal especializado. Além da conscientização dos profissionais em proporcionar um cuidado humanizado para essas gestantes, torna possíveis melhorias nos processos de referência e contrarreferência.

\section{Considerações Finais}

As gestantes encaminhadas para um serviço especializado trazem consigo preocupações em suas falas. Diante disso, são enfatizados sentimentos como insegurança, medo, angústia, decepção e ansiedade em virtude dos aspectos negativos encontrados no decorrer do pré-natal. Ainda assim, a experiência de ser mãe é um momento único e essa gestante precisa ser ouvida e acolhida.

Conforme os encaminhamentos pode-se observar as falhas dos profissionais de saúde, tanto da ESF quanto do serviço especializado visto que, a maioria das gestantes não compreendia a indicação para um acompanhamento de alto risco, além de que alguns encaminhamentos eram falsas indicações gerando atraso na referência de outrem.

Dentre as gestantes entrevistadas, a maioria estava na idade reprodutiva e já tiveram partos e gestações anteriores, o que supostamente transmite a ideia de possuírem uma experiência prévia sobre gestação. No entanto, cada gestação é única, vista de forma individualizada.

Os diagnósticos mais observados no presente estudo foram hipertensão e diabetes mellitus, retrato da realidade epidemiológica brasileira em que essas doenças têm como fatores de risco o estilo de vida contemporâneo.

$\mathrm{O}$ atendimento humanizado e acolhedor é indispensável na qualidade dos serviços prestados, fortalecendo o vínculo afetivo e fornecendo as devidas informações as gestantes satisfazendo as suas necessidades. Isso pode ser alcançado através da educação continuada, auxílio da instituição e ainda contribuição do município através cursos intensivos disponibilizados pela secretaria de saúde. 


\section{Agradecimentos}

Programa de Bolsas de Iniciação Científica da Pontifícia Universidade Católica de Minas Gerais- PROBIC- $2017 / 527$.

\section{Referências}

Antoniazzi, M. P., Siqueira, A. C., \& Farias, C, P. (2019). Aspectos psicológicos de uma gestação de alto risco em primigestas antes e depois do parto. Pensando familias, 23(2),191-207. http://pepsic.bvsalud.org/scielo.php?script=sci_arttext\&pid=S1679-494X2019000200015\&lng=pt\&tlng=pt

Bardin, L. (2010). Análise de conteúdo. Editora 70.

Brasil. Ministério da Saúde (2012a). Gestação de alto risco: manual técnico. Ministério da Saúde. http://bvsms.saude.gov.br/bvs/publicacoes/manual_tecnico_gestacao_alto_risco.pdf

Brasil. Ministério da Saúde (2012b). Conselho Nacional de Saúde. Resolução, n. 466/2012 - Normas para pesquisa envolvendo seres humanos. Brasília: Ministério da Saúde. http://conselho.saude.gov.br/web_comissoes/conep/aquivos/resolucoes/23_out_versao_final_196_ENCEP2012.pdf

Brasil. Ministério da Saúde. Sociedade Beneficente Israelita Brasileira Albert Einstein (2019). Nota técnica para organização da rede de atenção à saúde com foco na atenção primária à saúde e na atenção ambulatorial especializada - saúde da mulher na gestação, parto e puerpério. São Paulo: Hospital Israelita Albert Einstein. https://atencaobasica.saude.rs.gov.br/upload/arquivos/202001/03091259-nt-gestante-planificasus.pdf

Coelho, D. D. R., Souza, J. L. A., Torres, M. M. S. M. \& Drezett, J. (2017). Gravidez e maternidade tardia: sentimentos e vivências de mulheres em uma unidade de pré-natal de alto risco em Barreiras, Bahia. Rev Ciênc Saúde Oeste Baiano, 2(1):1-19. http://fasb.edu.br/revista/index.php/higia/article/view/145/202

Costa, L. D., Cura, C.C., Perondi, A.R., França, V.F. \& Bortoloti, D.S. (2016). Perfil epidemiológico de gestantes de alto risco. Cogitare Enferm, 21(2):01-08. http://dx.doi.org/10.5380/ce.v21i2.44192

Dias, E., Anjos, G., Alves, L., Pereira, S., \& Campos, L. (2018). Ações do enfermeiro no pré-natal e a importância atribuída pelas gestantes. Revista Sustinere, 6(1), 52-62. https://doi.org/10.12957/sustinere.2018.31722

Domingues, R. M. S. M., Viellas, E. F., Dias, M. A. B., Torres, J.A., Theme-Filha, M. M., Gama, S. G. N. et al. (2015). Adequação da assistência pré-natal segundo as características maternas no Brasil. Rev Panam Salud Pública, 37(3):140-7. https://scielosp.org/article/rpsp/2015.v37n3/140-147/pt/

Errico, L.S.P., Bicalho, P.G., Oliveira, T.C.F.L. \& Martins, E.F. The work of nurses in high-risk prenatal care from the perspective of basic human needs. Rev Bras Enferm. 2018;71(Suppl 3):1257-64. http://dx.doi.org/10.1590/0034-7167-2017-0328

Fernandes, J. A., Venâncio, S. I., Pasche, D. F., Silva, F. L. G., Aratani, N., Tanaka, O. Y. et al. (2020). Avaliação da atenção à gestação de alto risco em quatro metrópoles brasileiras. Cadernos de Saúde Pública, 36 (5): e00120519. https://doi.org/10.1590/0102-311X00120519

Gaíva, M. A. M., Palmeira, E. W. M. \& Mufato, L. F. (2017). Women's perception of prenatal and delivery care in cases of neonatal death. Esc Anna Nery, 21(4): e20170018. https://doi.org/10.1590/2177-9465-EAN-2017-0018

Grein, T. A. D., Nascimento, V. F., Hattori, T.Y., Terças, A. C. P. \& Borges, A. P. (2017). Saberes de puérperas sobre o planejamento reprodutivo. Semina Ciênc Biol Saúde, 38(2):145-54. https://doi.org/10.5433/1679-0367.2017v38n2p145

Jantsch, P. F., Carreno, I., Pozzobon, A., Adami, F. S., Leal, C. S., Mathias, T. C. S. et al. (2017). Principais características das gestantes de alto risco da região central do Rio Grande do Sul. Rev Dest Acad, 9:272-82. http://dx.doi.org/10.22410/issn.2176-3070.v9i3a2017.1534

Lee, S., Ayers, S. \& Holden, D. (2016). Risk perception and choice of place of birth in women with high risk pregnancies: A qualitative study. Midwifery, 12(38):49-54. https://doi.org/10.1016/j.midw.2016.03.008

Martins, A. C. S. \& Silva, L. S. (2018). Epidemiological profile of maternal mortality. Rev Bras Enferm, 71(Suppl 1):677-83. https://doi.org/10.1590/00347167-2017-0624

Martins, M. H. P. A., Ghersel, E. L. A. \& Ghersel, H. (2017). Identificação dos principais problemas em gestação de risco para nortear ações preventivas. Ciênc Saúde, 10(1):18-22. https://doi.org/10.15448/1983-652X.2017.1.23449

Menezes, J. J. S., Machado, S. L. da S., Galdino, C. V., Balbino, C. M., Silvino, Z. R., Santos, L. M. dos, \& Joaquim, F. L. (2020). Pré-natal de baixo risco: dificuldade da gestante na realização do pré-natal com o Enfermeiro. Research, Society and Development, 9(7), e912974497. https://doi.org/10.33448/rsdv9i7.4497

Oliveira, D. C. \& Mandú, E. N. T. (2015). Women with high-risk pregnancy: experiences and perceptions of needs and care. Esc Anna Nery - Rev Enferm, 19(1): 93-101. https://doi.org/10.5935/1414-8145.20150013

Pereira, D. O., Ferreira, T. L. S., Araújo, D. V., Melo, K.D. F., \& Andrade, F. B. (2018). Avaliação das consultas de pré-natal: adesão do pré-natal e complicações na saúde materno-infantil. Revista Ciência Plural, 3(3), 2-15. https://periodicos.ufrn.br/rcp/article/view/12891

Ragagnin, M. V., Marchioiri, M. R. C. T., Diaz, C. M. G., Nicolli, T., Pereira, S. B. \& Silva, L. D. (2017). Abordagem da equipe de enfermagem acerca do parto humanizado no pré-natal: uma revisão narrativa. Rev Pesqui Cuid Fundam Online, 9(4):1177-82. https://doi.org/10.9789/2175-5361.2017.v9i4.1177-1

Rodrigues, A. R. M., Dantas, S. L. C., Pereira, A. M. M., Silveira, M. A. M. \& Rodrigues, D. P. (2017). Gravidez de alto risco: análise dos determinantes de saúde. Sanare Sobral, 16(1):23-8. https://sanare.emnuvens.com.br/sanare/article/view/1135/620 
Research, Society and Development, v. 10, n. 9, e38310918295, 2021

(CC BY 4.0) | ISSN 2525-3409 | DOI: http://dx.doi.org/10.33448/rsd-v10i9.18295

Rodrigues, A. R. S., Barros, W.M. \& Soares, P. D. F. L. (2016). Reincidência da gravidez na adolescência: percepções das adolescentes. Enferm em Foco, 7(3/4):66-70. http://revista.cofen.gov.br/index.php/enfermagem/article/view/945/355

Salazar, C., Valério, E., Passos, E., Furasté, E. E., Oliveira, E. B., Arlindo, E. M. et al. (2016). Protocolos de encaminhamento para Obstetrícia (Pré- Natal de Alto Risco). https://www.ufrgs.br/telessauders/documentos/protocolos_resumos/protocolo_encaminhamento_obstetricia_TSRS_20160324.pdf

Saviani-Zeoti, F. \& Petean, E. B. L. (2015). Maternal-fetal attachment, anxiety, and depression in normal and high-risk pregnancies: A comparative study. Estud Psicol Camp, 32(4):675-83. https://doi.org/10.1590/0103-166X2015000400010

Silva, A.S.T., Souza, M.D., Oliveria, F.L.S., Furlan, C.S., Guerra, F.E.C. \& Buriola, A.A. (2020). Crenças e práticas espirituais/religiosas entre gestantes de alto risco. REPENF - Rev. Parana. Enferm. 3(1):46-54. http://seer.fafiman.br/index.php/REPEN/article/view/601

Taghizadeh, Z., Cheraghi, M.A., Kazemnejad, A., Pooralajal, J. \& Aghababaei, S. (2017). Difference in Perception of Pregnancy Risk in Two Maternal Age Groups. J Clin Diagn Res JCDR, 11(5):QC09-QC12. https://doi.org/10.7860/JCDR/2017/23661.9915

Universidade Estadual de Campinas. (2017). Pré-Natal e Puerpério Manual Técnico: manual de consulta rápida para os profissionais de saúde. São Paulo: Secretaria da Saúde do Governo do Estado de São Paulo. 123 p. http://www.saude.sp.gov.br/resources/ses/perfil/gestor/homepage/programa-defortalecimento-da-gestao-da-saude-no-estado-de-sao-paulo/consultas-publicas-manuais-da-linha-de-cuidado-da-gestante-parturiente-e-

puerpera/manual_de_consulta_rapida.pdf

Wilhelm, L. A., Alves, C. N., Demori, C. C., Silva, S. C., Meincke, S. M. K. \& Ressel, L. B. (2015). Feelings of women who experienced a high-risk pregnancy: a descriptive study. Online Braz J Nurs, 14(3):284-93. https://doi.org/10.17665/1676-4285.20155206 